\title{
2-D Reconstruction of Breast Image Using Forward- Backward Time-Stepping Method for Breast Tumour Detection
}

\author{
Marta A/P Elizabeth, Kismet Anak Hong Ping, \\ $\mathrm{Ng}$ Shi Wei, Wan Azlan bin Wan Zainal Abidin, \\ Thelaha bin Masri, Al-Khalid bin Othman \\ Department of Electronic Engineering, \\ Universiti Malaysia Sarawak, Kota Samarahan, \\ Sarawak, Malaysia \\ 11021798@siswa.unimas.my, hpkismet@feng.unimas.my
}

\author{
Toshifumi Moriyama, Takashi Takenaka \\ Department of Electrical and Electronic Engineering \\ Nagasaki University, \\ Nagasaki, Japan \\ t-moriya@nagasaki-u.ac.jp, takenaka@nagasaki-u.ac.jp
}

\begin{abstract}
In this paper, time-domain inverse-scattering technique referred to as Forward-Backward Time-Stepping (FBTS) method is applied to microwave imaging breast cancer detection. The FBTS algorithm is implemented in $\mathrm{C}++$ language executed in a single computer by developing the time domain form of the FDTD. The ability to detect as small as $4 \mathrm{~mm}$ of the embedded tumour and reconstructing the image in twodimensional with significant contrast in the dielectric properties of normal breast tissue and malignant tumour is presented in this paper.
\end{abstract}

Index Terms-Breast cancer detection, microwave imaging, inverse scattering, tumour.

\section{INTRODUCTION}

Breast cancer still remains the most common cause of death among women. X-ray mammography and ultrasound scanning are two common methods for detecting breast cancer at early stage. However, these two methods do have limitations. Mammography is the most effective imaging method of breast cancer, however in order to gain the image, the process could be painful and uncomfortable to the patient. The existence of false negative (undetected cancers) or false positive (lump but not cancer) result rate is relatively high particularly in pre-menopausal women where increased breast density can obscure impalpable lesions [1]. As reported in [2], the mammographic screening has missed up to $15 \%$ of tumours and is less effective for detecting tumour in a dense breast (particularly for women less than 50 years old).

The last two decades have shown a significant increase in research pertaining early breast cancer screening method using microwave imaging. The significant contrast in the dielectric properties of normal breast tissue and malignant tumours provides a valid reason for researchers to continue in the development of microwave based imaging [2-8].

Recent investigations have been made in constructing a better quality image and in providing useful information utilizing microwave imaging such as microwave imaging via space-time beamforming [5-6]. Another method was reported in [7], multistatic adaptive microwave imaging promising higher resolution, lower sidelobes, better noise and interference rejection capability.

There are two common techniques for microwave screening techniques, inverse scattering and ultra-wideband radar based. The former approaches has the ability to reconstruct quantitative images with the internal relative permittivity $\left(\mathcal{E}_{r}\right)$ and conductivity $(\sigma)$ composition of breast. The time domain scattering data contains more information compared to a single frequency scattering data. Therefore, electromagnetic imaging in the time domain has the potential of reconstructing electrical parameter profiles more accurately. In our study, we investigated a technique for breast cancer detection and the analysis which combines the advantages of these two approaches [8].

Microwave screening for breast cancer detection methods have been found to be more comfortable to the patient, low cost of microwave scanning system and safe [9].

In our research, we proposed Forward-Backward TimeStepping (FBTS) method formulated in the time domain to reconstruct the dielectric properties of the present malignant and surrounding breast tissue. By utilizing finite-difference time-domain (FDTD) method, the FBTS technique has the potential to reconstruct images that provide useful quantitative information about the location, size, shape and the internal composition of the malignancies [2-14]. The ability of FBTS to detect the tumour embedded in the fibroglandular as small as $4 \mathrm{~mm}$ in the breast model will be demonstrated in this paper.

\section{METHODS}

\section{A. Inverse Scattering Techniques}

We consider inverse scattering problem where the electrical property distributions within a target object are estimated from scattering time domain data. The breast is surrounded by eight antennas as illustrated in Figure 1. Each antenna takes turn to transmit a microwave pulse while the rest of antennas collect 